\title{
Existence of Positive Solutions for Nonlocal Fourth-Order Boundary Value Problem with Variable Parameter
}

\author{
Xiaoling Han, Hongliang Gao, and Jia Xu \\ Department of Mathematics, Northwest Normal University, Lanzhou 730070, China \\ Correspondence should be addressed to Xiaoling Han, hanxiaoling@nwnu.edu.cn \\ Received 26 November 2010; Accepted 14 January 2011 \\ Academic Editor: M. Furi
}

Copyright (C) 2011 Xiaoling Han et al. This is an open access article distributed under the Creative Commons Attribution License, which permits unrestricted use, distribution, and reproduction in any medium, provided the original work is properly cited.

By using the Krasnoselskii's fixed point theorem and operator spectral theorem, the existence of positive solutions for the nonlocal fourth-order boundary value problem with variable parameter $u^{(4)}(t)+B(t) u^{\prime \prime}(t)=\lambda f\left(t, u(t), u^{\prime \prime}(t)\right), 0<t<1, u(0)=u(1)=\int_{0}^{1} p(s) u(s) d s, u^{\prime \prime}(0)=u^{\prime \prime}(1)=$ $\int_{0}^{1} q(s) u^{\prime \prime}(s) d s$ is considered, where $p, q \in L^{1}[0,1], \lambda>0$ is a parameter, and $B \in C[0,1], f \in$ $C([0,1] \times[0, \infty) \times(-\infty, 0],[0, \infty))$.

\section{Introduction}

The existence of positive solutions for nonlinear fourth-order multipoint boundary value problems has been studied by many authors using nonlinear alternatives of Leray-Schauder, the fixed point theory, and the method of upper and lower solutions (see, e.g., [1-15] and references therein). The multipoint boundary value problem is in fact a special case of the boundary value problem with integral boundary conditions.

Recently, Bai [16] studied the existence of positive solutions of nonlocal fourth-order boundary value problem

$$
\begin{gathered}
u^{(4)}(t)+\beta u^{\prime \prime}(t)=\lambda f\left(t, u(t), u^{\prime \prime}(t)\right), \quad 0<t<1, \\
u(0)=u(1)=\int_{0}^{1} p(s) u(s) d s, \\
u^{\prime \prime}(0)=u^{\prime \prime}(1)=\int_{0}^{1} q(s) u^{\prime \prime}(s) d s .
\end{gathered}
$$


under the assumption:

$$
\begin{aligned}
& \text { (A1) } \lambda>0 \text { and } 0<\beta<\pi^{2}, \\
& \text { (A2) } f \in C([0,1] \times[0, \infty) \times(-\infty, 0],[0, \infty)), p, q \in L^{1}[0,1], p(s) \geq 0, q(s) \geq 0, \int_{0}^{1} p(s) d s<1, \\
& \int_{0}^{1} q(s) \sin \sqrt{\beta} s d s+\int_{0}^{1} q(s) \sin \sqrt{\beta}(1-s) d s<\sin \sqrt{\beta} .
\end{aligned}
$$

In this paper, we study the above generalizing form with variable parameters BVP

$$
\begin{gathered}
u^{(4)}(t)+B(t) u^{\prime \prime}(t)=\lambda f\left(t, u(t), u^{\prime \prime}(t)\right), \quad 0<t<1, \\
u(0)=u(1)=\int_{0}^{1} p(s) u(s) d s, \\
u^{\prime \prime}(0)=u^{\prime \prime}(1)=\int_{0}^{1} q(s) u^{\prime \prime}(s) d s,
\end{gathered}
$$

where $B \in C[0,1], \lambda>0$ is a parameter.

Obviously, BVP(1.1) can be regarded as the special case of $\operatorname{BVP}(1.2)$ with $B(t)=\beta$. Since the parameters $B(t)$ is variable, we cannot expect to transform directly $\mathrm{BVP}(1.2)$ into an integral equation as in [16]. We will apply the cone fixed point theory, combining with the operator spectra theorem to establish the existence of positive solutions of BVP(1.2). Our results generalize the main result in [16].

Let $\beta=\inf _{t \in[0,1]} B(t)$, and we assume that the following conditions hold throughout the paper:

(H1) $B \in C[0,1]$ and $0<\beta<\pi^{2}$,

(H2) $f \in C([0,1] \times[0, \infty) \times(-\infty, 0],[0, \infty)), p, q \in L^{1}[0,1], p(s) \geq 0, q(s) \geq 0$ and $\int_{0}^{1} p(s) d s<1, \int_{0}^{1} q(s) \sin \sqrt{\beta} s d s+\int_{0}^{1} q(s) \sin \sqrt{\beta}(1-s) d s<\sin \sqrt{\beta}$.

\section{The Preliminary Lemmas}

Set $\lambda_{1}=0,-\pi^{2}<\lambda_{2}=-\beta<0$ and

$$
\delta_{1}=1-\int_{0}^{1} p(s) d s, \quad \delta_{2}=\sin \sqrt{\beta}-\int_{0}^{1} q(s) \sin \sqrt{\beta} s d s-\int_{0}^{1} q(s) \sin \sqrt{\beta}(1-s) d s .
$$

By (H1), (H2), we get $\delta_{i} \neq 0, i=1,2$. Denote by $K_{1}(t, s)$ the Green's function of the problem

$$
\begin{gathered}
-u^{\prime \prime}(t)+\lambda_{1} u(t)=0, \quad 0<t<1, \\
u(0)=u(1)=\int_{0}^{1} p(s) u(s) d s
\end{gathered}
$$


and $K_{2}(t, s)$ the Green's function of the problem

$$
\begin{gathered}
-u^{\prime \prime}(t)+\lambda_{2} u(t)=0, \quad 0<t<1, \\
u(0)=u(1)=\int_{0}^{1} q(s) u(s) d s .
\end{gathered}
$$

Then, carefully calculation yield

$$
\begin{gathered}
K_{1}(t, s)=G_{1}(t, s)+\rho_{1} \int_{0}^{1} G_{1}(s, x) p(x) d x, \\
K_{2}(t, s)=G_{2}(t, s)+\rho_{2}(t) \int_{0}^{1} G_{2}(s, x) q(x) d x, \\
G_{1}(t, s)= \begin{cases}t(1-s), \quad 0 \leq t \leq s \leq 1, \\
s(1-t), \quad 0 \leq s \leq t \leq 1,\end{cases} \\
G_{2}(t, s)=\left\{\begin{array}{l}
\frac{\sin \sqrt{\beta} t \sin \sqrt{\beta}(1-s)}{\sqrt{\beta} \sin \sqrt{\beta}}, \quad 0 \leq t \leq s \leq 1, \\
\frac{\sin \sqrt{\beta} s \sin \sqrt{\beta}(1-t)}{\sqrt{\beta} \sin \sqrt{\beta}}, \quad 0 \leq s \leq t \leq 1, \\
\rho_{1}=\frac{1}{\delta_{1}}, \quad \begin{array}{c}
\sin \sqrt{\beta} t+\sin \sqrt{\beta}(1-t) \\
\rho_{2}(t)=\frac{\delta_{2}}{}
\end{array}
\end{array}\right.
\end{gathered}
$$

Lemma 2.1 (see [16]). Suppose that (A1), (A2) hold. Then, for any $h \in C[0,1]$, u solves the problem

$$
\begin{gathered}
u^{(4)}(t)+\beta u^{\prime \prime}(t)=h(t), \quad 0<t<1 \\
u(0)=u(1)=\int_{0}^{1} p(s) u(s) d s \\
u^{\prime \prime}(0)=u^{\prime \prime}(1)=\int_{0}^{1} q(s) u^{\prime \prime}(s) d s
\end{gathered}
$$

if and only if $u(t)=\int_{0}^{1} \int_{0}^{1} K_{1}(t, s) K_{2}(s, \tau) h(\tau) d \tau d s$.

Let $Y=C[0,1], Y_{+}=\{u \in Y: u(t) \geq 0, t \in[0,1]\}$, and $\|u\|_{0}=\max _{0 \leq t \leq 1}|u(t)|$, for $u \in Y$. $X=\left\{u \in C^{2}[0,1]: u(0)=u(1)=\int_{0}^{1} p(s) u(s) d s, u^{\prime \prime}(0)=u^{\prime \prime}(1)=\int_{0}^{1} q(s) u^{\prime \prime}(s) d s\right\},\|u\|_{1}=\left\|u^{\prime \prime}\right\|_{0}$, $\|u\|_{2}=\|u\|_{0}+\|u\|_{1}$, for $u \in X$.

It is easy to show that $\|u\|_{1},\|u\|_{2}$ are norms on $X$. 
Lemma 2.2 (see [16]). $\|\cdot\|_{1} \leq\|\cdot\|_{2} \leq\left(1+\delta_{1}\right)\|\cdot\|_{1}$ and $\left(X,\|\cdot\|_{2}\right)$ is a Banach space.

Lemma 2.3 (see [5]). Assume that (A1), (A2) hold. Then,

(i) $K_{i}(t, s) \geq 0$, for $t, s \in[0,1], i=1,2 ; K_{i}(t, s)>0$, for $t, s \in(0,1), i=1,2$,

(ii) $G_{i}(t, s) \geq b_{i} G_{i}(t, t) G_{i}(s, s), G_{i}(t, s) \leq C_{i} G_{i}(s, s)$ for $t, s \in[0,1], i=1,2$,

where $C_{1}=1, b_{1}=1 ; C_{2}=1 / \sin \sqrt{\beta}, b_{2}=\sqrt{\beta} \sin \sqrt{\beta}$.

Denote

$$
\begin{gathered}
d_{i}=\min _{1 / 4 \leq t \leq 3 / 4} b_{i} G_{i}(t, t) \quad(i=1,2), \\
\xi=\frac{\min _{1 / 4 \leq t \leq 3 / 4} \rho_{2}(t)}{\max _{1 / 4 \leq t \leq 3 / 4} \rho_{2}(t)} \\
D_{i}=\max _{t \in[0,1]} \int_{0}^{1} K_{i}(t, s) d s \quad(i=1,2) .
\end{gathered}
$$

Computations yield the following results.

Lemma 2.4 (see [3]). $D_{i}^{1}=\max _{t \in[0,1]} \int_{0}^{1} G_{i}(t, s) d s>0(i=1,2)$

(i) when $\lambda_{i}>0, D_{i}^{1}=\left(1 / \lambda_{i}\right)\left(1-1 / \cos \left(\omega_{i} / 2\right)\right)$,

(ii) when $\lambda_{i}=0, D_{i}^{1}=1 / 8$,

(iii) when $-\pi^{2}<\lambda_{i}<0, D_{i}^{1}=\left(1 / \lambda_{i}\right)\left(1-1 / \cos \left(\omega_{i} / 2\right)\right)$.

Lemma 2.5 (see [16]). Suppose that (A1), (A2) hold and $\rho_{2}(t), d_{i}, \xi$ are given as above. Then,

(i) $\max _{t \in[0,1]} \rho_{2}(t)=\rho_{2}(1 / 2)$,

(ii) $0<d_{i}<1,0<\xi<1$.

By Lemmas 2.4 and 2.5, $D_{2}=\max _{t \in[0,1]} \int_{0}^{1} K_{2}(1 / 2, s) d s$.

Take $\theta=\min \left\{d_{1}, d_{2} \xi / C_{2}\right\}$, by Lemma $2.5,0<\theta<1$.

Define

$$
\begin{aligned}
& (T h)(t)=\int_{0}^{1} \int_{0}^{1} K_{1}(t, s) K_{2}(s, \tau) h(\tau) d \tau d s, \quad t \in[0,1] \\
& (A h)(t)=(T h)^{\prime \prime}(t)=-\int_{0}^{1} K_{2}(t, \tau) h(\tau) d \tau, \quad t \in[0,1]
\end{aligned}
$$

Lemma 2.6. $T: Y \rightarrow\left(X,\|\cdot\|_{2}\right)$ is completely continuous, and $\|T\| \leq D_{2}$.

Proof. It is similar to Lemma 6 of [3], so we omit it. 
Lemma 2.7 (see [17]). Let $E$ be a Banach space, $P \subseteq E$ a cone, and $\Omega_{1}, \Omega_{2}$ be two bounded open sets of $E$ with $0 \in \Omega_{1} \subset \bar{\Omega}_{1} \subset \Omega_{2}$. Suppose that $A: P \cap\left(\bar{\Omega}_{2} \backslash \Omega_{1}\right) \rightarrow P$ is a completely continuous operator such that either

(i) $\|A x\| \leq\|x\|, x \in P \cap \partial \Omega_{1}$ and $\|A x\| \geq\|x\|, x \in P \cap \partial \Omega_{2}$, or

(ii) $\|A x\| \geq\|x\|, x \in P \cap \partial \Omega_{1}$ and $\|A x\| \leq\|x\|, x \in P \cap \partial \Omega_{2}$

holds. Then, $A$ has a fixed point in $P \cap\left(\bar{\Omega}_{2} \backslash \Omega_{1}\right)$.

\section{The Main Results}

Suppose that $K_{1}, K_{2}, G_{2}, \rho_{2}, C_{2}, \theta$, and $D_{2}$, are defined as in Section 2, we introduce some notations as follows:

$$
\begin{gathered}
A=\int_{0}^{1} \int_{0}^{1} K_{1}(s, s) K_{2}(s, \tau) d \tau d s, \quad B=\int_{0}^{1}\left[G_{2}(s, s)+\rho_{2}\left(\frac{1}{2}\right) \int_{0}^{1} G_{2}(s, x) q(x) d x\right] d s, \\
K=\sup _{t \in[0,1]}[B(t)-\beta], \quad L=D_{2} K, \quad \eta_{0}=\frac{1-L}{A+C_{2} B}, \quad \eta_{1}=\frac{1}{\theta \int_{1 / 4}^{3 / 4} K_{2}(1 / 2, \tau) d \tau}, \\
\bar{f}_{0}=\limsup _{|u|+|v| \rightarrow 0} \max _{t \in[0,1]} \frac{f(t, u, v)}{|u|+|v|}, \quad \underline{f}_{0}=\lim _{|u|+|v| \rightarrow 0} \min _{t \in[1 / 4,3 / 4]} \frac{f(t, u, v)}{|u|+|v|} \\
\bar{f}_{\infty}=\limsup _{|u|+|v| \rightarrow+\infty} \max _{t \in[0,1]} \frac{f(t, u, v)}{|u|+|v|}, \quad \underline{f}_{\infty}=\liminf _{|u|+|v| \rightarrow+\infty} \min _{t \in[1 / 4,3 / 4]} \frac{f(t, u, v)}{|u|+|v|} .
\end{gathered}
$$

Theorem 3.1. Assume that (H1), (H2) hold and $L=D_{2} K<1$. Then BVP(1.2) has at least one positive solution if one of the following cases holds:

(i) $\bar{f}_{0}<(1 / \lambda) \eta_{0}, \underline{f}_{\infty}>(1 / \lambda) \eta_{1}$

(ii) $\underline{f}_{0}>(1 / \lambda) \eta_{1}, \bar{f}_{\infty}<(1 / \lambda) \eta_{0}$.

Proof. For any $h \in Y$, consider the following BVP:

$$
\begin{gathered}
u^{(4)}(t)+B(t) u^{\prime \prime}(t)=h(t), \quad 0<t<1 \\
u(0)=u(1)=\int_{0}^{1} p(s) u(s) d s \\
u^{\prime \prime}(0)=u^{\prime \prime}(1)=\int_{0}^{1} q(s) u^{\prime \prime}(s) d s .
\end{gathered}
$$


It is easy to see that the above question is equivalent to the following question:

$$
\begin{aligned}
u^{(4)}(t)+\beta u^{\prime \prime}(t) & =-(B(t)-\beta) u^{\prime \prime}(t)+h(t), \quad 0<t<1, \\
u(0) & =u(1)=\int_{0}^{1} p(s) u(s) d s, \\
u^{\prime \prime}(0) & =u^{\prime \prime}(1)=\int_{0}^{1} q(s) u^{\prime \prime}(s) d s .
\end{aligned}
$$

For any $v \in X$, let $G v=-(B(t)-\beta) v^{\prime \prime}$. Obviously, the operator $G: X \rightarrow Y$ is linear. By Lemma 2.2, for all $v \in X, t \in[0,1],|(G v)(t)| \leq(B(t)-\beta)\|v\|_{1} \leq K\|v\|_{1} \leq K\|v\|_{2}$. Hence $\|G v\|_{0} \leq K\|v\|_{2}$, and so $\|G\| \leq K$. On the other hand, $u \in C^{2}[0,1] \cap C^{4}(0,1)$ is a solution of (3.3) if and only if $u \in X$ satisfies $u=T(G u+h)$, that is,

$$
u \in X, \quad(I-T G) u=T h .
$$

Owing to $G: X \rightarrow Y$ and $T: Y \rightarrow X$, the operator $I-T G$ maps $X$ into $X$. From $\|T\| \leq D_{2}$ (by Lemma 2.6) together with $\|G\| \leq K$ and condition $L<1$, applying operator spectral theorem, we have that the $(I-T G)^{-1}$ exists and is bounded. Let $H=(I-T G)^{-1} T$, then (3.4) is equivalent to $u=H h$. By the Neumann expansion formula, $H$ can be expressed by

$$
H=\left(I+T G+\cdots+(T G)^{n}+\cdots\right) T=T+(T G) T+\cdots+(T G)^{n} T+\cdots
$$

The complete continuity of $T$ with the continuity of $(I-T G)^{-1}$ yields that the operator $H$ : $Y \rightarrow X$ is completely continuous. For all $h \in Y_{+}$, let $u=T h$, then $u \in X \cap Y_{+}$, and $u^{\prime \prime}<0$. So, we have $(G u)(t)=-(B(t)-\beta) u^{\prime \prime}(t) \geq 0, t \in[0,1]$. Hence,

$$
\forall h \in Y_{+}, \quad(G T h)(t) \geq 0, \quad t \in[0,1]
$$

and so $(T G)(T h)(t)=T(G T h)(t) \geq 0, t \in[0,1]$.

Assume that for all $h \in Y_{+},(T G)^{k}(T h)(t) \geq 0, t \in[0,1]$, let $h_{1}=G T h$, by (3.6) we have $h_{1} \in Y_{+}$, and so $(T G)^{k+1}(T h)(t)=(T G)^{k}(T G T h)(t)=(T G)^{k}\left(T h_{1}\right)(t) \geq 0, t \in[0,1]$. Thus by induction, it follows that $(T G)^{n}(T h)(t) \geq 0$, for all $n \geq 1, h \in Y_{+}, t \in[0,1]$. By (3.5), for all $h \in Y_{+}$, we have

$$
\begin{aligned}
(H h)(t) & =(T h)(t)+(T G)(T h)(t)+\cdots+(T G)^{n}(T h)(t)+\cdots \geq(T h)(t), \quad t \in[0,1], \\
(H h)^{\prime \prime}(t) & =(A h)(t)+(A G)(T h)(t)+\cdots+\left(A G(T G)^{n-1}\right)(T h)(t)+\cdots \\
& \leq(A h)(t)=(T h)^{\prime \prime}(t) \leq 0, \quad t \in[0,1]
\end{aligned}
$$

and so $H: Y_{+} \rightarrow Y_{+} \cap X$. 
Fixed Point Theory and Applications

On the other hand, for all $h \in Y_{+}$, we have

$$
\begin{aligned}
(H h)(t) & \leq(T h)(t)+|T G|(T h)(t)+\cdots+|T G|^{n}(T h)(t)+\cdots \\
& \leq\left(1+L+\cdots+L_{n}+\cdots\right)(T h)(t) \\
& =\frac{1}{1-L}(T h)(t) \quad t \in[0,1], \\
\left|(H h)^{\prime \prime}(t)\right| & \leq|(A h)(t)|+|(A G)(T h)(t)|+\cdots+\left|\left(A G(T G)^{n-1}\right)(T h)(t)\right|+\cdots \\
& \leq|(A h)(t)|+L|(A h)(t)|+\cdots+L^{n}|(A h)(t)|+\cdots \\
& =\left(1+L+\cdots+L_{n}+\cdots\right)|(A h)(t)| \\
& =\frac{1}{1-L}\left|(T h)^{\prime \prime}(t)\right| \quad t \in[0,1], \\
& \|H h\|_{0} \geq\|T h\|_{0}, \quad\|H h\|_{0} \leq \frac{1}{1-L}\|T h\|_{0}, \\
& \|H h\|_{1} \geq\|T h\|_{1}, \quad\|H h\|_{1} \leq \frac{1}{1-L}\|T h\|_{1} .
\end{aligned}
$$

For any $u \in Y_{+}$, define $F u=\lambda f\left(t, u, u^{\prime \prime}\right)$. By (H1) and (H2), we have that $F: Y_{+} \rightarrow Y_{+}$is continuous. It is easy to see that $u \in C^{2}[0,1] \cap C^{4}(0,1)$ being a positive solution of $\operatorname{BVP}(1.2)$ is equivalent to $u \in Y_{+}$being a nonzero solution equation as follows:

$$
u=H F u \text {. }
$$

Let $Q=H F$. Obviously, $Q: Y_{+} \rightarrow Y_{+}$is completely continuous. We next show that the operator $Q$ has a nonzero fixed point in $Y_{+}$. Let

$$
P=\left\{u \in X: u \geq 0, u^{\prime \prime} \leq 0, \min _{1 / 4 \leq t \leq 3 / 4} u(t) \geq(1-L) d_{1}\|u\|_{0}, \max _{1 / 4 \leq t \leq 3 / 4} u^{\prime \prime}(t) \leq-(1-L) \frac{d_{2} \xi}{C_{2}}\left\|u^{\prime \prime}\right\|_{0}\right\} .
$$

It is easy to know that $P$ is a cone in $X, P \subset Y_{+}$. Now, we show $Q P \subset P$.

For $h \in Y_{+}$, by (2.7), there is $T h \geq 0,(T h)^{\prime \prime} \leq 0$. Hence, by (3.7), $Q u \geq 0,(Q u)^{\prime \prime} \leq 0, u \in$ $P$. By proof of Lemma 2.5 in [16],

$$
\min _{1 / 4 \leq t \leq 3 / 4}(T h)(t) \geq d_{1}\|T h\|_{0}, \quad \max _{1 / 4 \leq t \leq 3 / 4}(T h)^{\prime \prime}(t) \leq-\frac{d_{2} \xi}{C_{2}}\left\|(T h)^{\prime \prime}\right\|_{0} .
$$


By (3.7) and (3.10),

$$
\begin{gathered}
\min _{1 / 4 \leq t \leq 3 / 4}(Q u)(t) \geq \min _{1 / 4 \leq t \leq 3 / 4}(T F u)(t) \geq d_{1}\|T F u\|_{0} \geq(1-L) d_{1}\|Q u\|_{0}, \\
\max _{1 / 4 \leq t \leq 3 / 4}(Q u)^{\prime \prime}(t) \leq \max _{1 / 4 \leq t \leq 3 / 4}(T F u)^{\prime \prime}(t) \leq-\frac{d_{2} \xi}{C_{2}}\left\|(T F u)^{\prime \prime}\right\|_{0} \leq-(1-L) \frac{d_{2} \xi}{C_{2}}\left\|(Q u)^{\prime \prime}\right\|_{0} .
\end{gathered}
$$

Thus $Q P \subset P$.

(i) Since $\bar{f}_{0}<(1 / \lambda) \eta_{0}$, by the definition of $\bar{f}_{0}$, there exists $r_{1}>0$ such that

$$
\max _{0 \leq t \leq 1,|u(t)|+\left|u^{\prime \prime}(t)\right| \leq r_{1}} f\left(t, u(t), u^{\prime \prime}(t)\right) \leq \frac{r_{1}}{\lambda} \eta_{0} .
$$

Let $\Omega_{r_{1}}=\left\{u \in P:\|u\|_{2}<r_{1}\right\}$, one has

$$
f\left(t, u(t), u^{\prime \prime}(t)\right) \leq \frac{r_{1}}{\lambda} \eta_{0}, \quad u \in \partial \Omega_{r_{1}}, t \in[0,1] .
$$

So, by (3.10), we get

$$
\begin{aligned}
\|Q u\|_{0}=\|H F u\|_{0} & \leq \frac{1}{1-L}\|T F u\|_{0} \\
& =\frac{\lambda}{1-L}\left\|\int_{0}^{1} \int_{0}^{1} K_{1}(t, s) K_{2}(s, \tau) f\left(\tau, u(\tau), u^{\prime \prime}(\tau)\right) d \tau d s\right\|_{0} \\
& \leq \frac{r_{1} \eta_{0}}{1-L} \int_{0}^{1} \int_{0}^{1} K_{1}(s, s) K_{2}(s, \tau) d \tau d s \leq \frac{A \eta_{0} r_{1}}{1-L}, \\
\|Q u\|_{1}=\|H F u\|_{1} & \leq \frac{1}{1-L}\|T F u\|_{1} \\
& \leq \lambda C_{2} \frac{1}{1-L} \int_{0}^{1}\left[G_{2}(\tau, \tau)+\rho_{2}\left(\frac{1}{2}\right) \int_{0}^{1} G_{2}(\tau, x) q(x) d x\right] f\left(\tau, u(\tau), u^{\prime \prime}(\tau)\right) d \tau \\
& \leq \frac{C_{2} B \eta_{0} r_{1}}{1-L} .
\end{aligned}
$$

Hence, for $u \in \partial \Omega_{r_{1}}$,

$$
\|Q u\|_{2}=\|H F u\|_{2} \leq \frac{1}{1-L}\|T F u\|_{2} \leq \frac{\left(A+B C_{2}\right) \eta_{0} r_{1}}{1-L}=r_{1}=\|u\|_{2} .
$$


On the other hand, since $\underline{f}_{\infty}>(1 / \lambda) \eta_{1}$, there exists $r_{2}^{\prime}>r_{1}>0$ such that

$$
\min _{1 / 4 \leq t \leq 3 / 4, \theta\left(|u(t)|+\left|u^{\prime \prime}(t)\right|\right) \geq r_{2}^{\prime}} \frac{f\left(t, u(t), u^{\prime \prime}(t)\right)}{|u(t)|+\left|u^{\prime \prime}(t)\right|} \geq \frac{1}{\lambda} \eta_{1}
$$

Choose $r_{2}>(1 / \theta) r_{2}^{\prime}$, let $\Omega_{r_{2}}=\left\{u \in P:\|u\|_{2}<r_{2}\right\}$. For $u \in \partial \Omega_{r_{2}}, t \in[1 / 4,3 / 4]$, there is $r_{2}^{\prime} \leq \theta r_{2} \leq|u(t)|+\left|u^{\prime \prime}(t)\right| \leq r_{2}$. Thus,

$$
\begin{gathered}
f\left(t, u(t), u^{\prime \prime}(t)\right) \geq \frac{\theta r_{2}}{\lambda} \eta_{1}, \quad u \in \partial \Omega_{r_{2}}, t \in\left[\frac{1}{4}, \frac{3}{4}\right] . \\
\left|(T F u)^{\prime \prime}\left(\frac{1}{2}\right)\right|=\lambda \int_{0}^{1} K_{2}\left(\frac{1}{2}, \tau\right) f\left(\tau, u(\tau), u^{\prime \prime}(\tau)\right) d \tau \\
\geq \lambda \int_{1 / 4}^{3 / 4} K_{2}\left(\frac{1}{2}, \tau\right) f\left(\tau, u(\tau), u^{\prime \prime}(\tau)\right) d \tau \geq \eta_{1} \theta r_{2} \int_{1 / 4}^{3 / 4} K_{2}\left(\frac{1}{2}, \tau\right) d \tau=r_{2} .
\end{gathered}
$$

Hence, for $u \in \Omega_{r_{2}}$,

$$
\|Q u\|_{2} \geq\|T F u\|_{2} \geq\left|(T F u)^{\prime \prime}\left(\frac{1}{2}\right)\right| \geq r_{2}=\|u\|_{2}
$$

By the use of the Krasnoselskii's fixed point theorem, we know there exists $u_{0} \in \bar{\Omega}_{2} \backslash \Omega_{1}$ such that $Q u_{0}=u_{0}$, namely, $u_{0}$ is a solution of (1.2) and satisfied $u_{0} \geq 0, u_{0}^{\prime \prime} \leq 0, r_{1} \leq\left\|u_{0}\right\|_{2} \leq r_{2}$.

(ii) The proof is similar to (i), so we omit it.

Corollary 3.2. Assume that (H1), (H2) hold, and $L<1$. Then that (1.2) has at least two positive solution, if $f$ satisfy

(i) $\bar{f}_{0}<(1 / \lambda) \eta_{0}, \bar{f}_{\infty}<(1 / \lambda) \eta_{0}$,

(ii) There exists $R_{0}>0$ such that $f(t, u, v) \geq\left(\theta R_{0} / \lambda\right) \eta_{1}$, for $t \in[1 / 4,3 / 4],|u|+|v| \geq \theta R_{0}$.

Proof. By the proof of Theorem 3.1, we know that (1) from the condition $\bar{f}_{0}<(1 / \lambda) \eta_{0}$, there exists $\Omega_{r_{1}}=\left\{u \in P:\|u\|_{2}<r_{1}\right\}$, such that $\|Q u\|_{2} \leq\|u\|_{2}, u \in \partial \Omega_{r_{1}}$, (2) from the condition $\bar{f}_{\infty}<(1 / \lambda) \eta_{0}$, there exists $\Omega_{r_{2}}=\left\{u \in P:\|u\|_{2}<r_{2}\right\}, r_{2}>r_{1}$, such that $\|Q u\|_{2} \leq\|u\|_{2}, u \in \partial \Omega_{r_{2}}$, (3) from the condition (ii), there exists $\Omega_{r_{3}}=\left\{u \in P:\|u\|_{2}<r_{3}\right\}, r_{2}>r_{3}>r_{1}$, such that $\|Q u\|_{2} \geq\|u\|_{2}, u \in \partial \Omega_{r_{3}}$. By the use of Krasnoselskii's fixed point theorem, it is easy to know that (1.2) has at least two positive solutions.

Corollary 3.3. Assume (H1), (H2) hold, and L $<1$. Then problem (1.2) has at least two positive solution, if $f$ satisfy

(i) $\underline{f}_{0}>(1 / \lambda) \eta_{1}, \underline{f}_{\infty}>(1 / \lambda) \eta_{1}$

(ii) There exists $R_{0}>0$ such that $f(t, u, v) \leq\left(\theta R_{0} / \lambda\right) \eta_{0}$, for $t \in[0,1],|u|+|v| \leq R_{0}$.

Proof. The proof is similar to Corollary 3.2, so we omit it. 
Example 3.4. Consider the following boundary value problem

$$
\begin{gathered}
u^{(4)}(t)+\left(\frac{\pi^{2}}{4}+t\right) u^{\prime \prime}(t)=\pi^{2}\left[18\left(u(t)-u^{\prime \prime}(t)\right)-17.9 \sin \left(u(t)-u^{\prime \prime}(t)\right)\right], \quad 0<t<1, \\
u(0)=u(1)=\int_{0}^{1} s u(s) d s, \\
u^{\prime \prime}(0)=u^{\prime \prime}(1)=0 .
\end{gathered}
$$

In this problem, we know that $B(t)=\pi^{2} / 4+t, p(t)=t, q(t)=0, \lambda=\pi^{2}$, then we can get $C_{1}=1, C_{2}=1, \rho_{1}=1, \rho_{2}=\sqrt{2}, \beta=\pi^{2} / 4, K=1, D_{2}=4(\sqrt{2}-1) / \pi^{2}$. Further more, we obtain $A=\left(48-13 \pi^{2}\right) / \pi^{3}, B=2 / \pi^{2}$, then $\eta_{0}=(1-L) \pi^{3} /(48-11 \pi), \eta_{1}=4 \pi^{2} / \sqrt{2} \cos (\pi / 8)-1$, so

$$
\bar{f}_{0}=0.1<\frac{1}{\pi^{2}} \eta_{0} \approx 0.19, \quad \underline{f}_{\infty}=18>\frac{1}{\pi^{2}} \eta_{1} \approx 13.3 .
$$

Thus, $B(t), p(t), q(t)$, and $f$ satisfy the conditions of Theorem 3.1, and there exists at least a positive solution of the above problem.

\section{Acknowledgments}

This work is sponsored by the NSFC (no. 11061030), NSFC (no. 11026060), and nwnu-kjcxgc03-69, 03-61.

\section{References}

[1] Z. Bai, "The method of lower and upper solutions for a bending of an elastic beam equation," Journal of Mathematical Analysis and Applications, vol. 248, no. 1, pp. 195-202, 2000.

[2] Z. Bai, "The upper and lower solution method for some fourth-order boundary value problems," Nonlinear Analysis. Theory, Methods E Applications, vol. 67, no. 6, pp. 1704-1709, 2007.

[3] G. Chai, "Existence of positive solutions for fourth-order boundary value problem with variable parameters," Nonlinear Analysis. Theory, Methods \& Applications, vol. 66, no. 4, pp. 870-880, 2007.

[4] H. Feng, D. Ji, and W. Ge, "Existence and uniqueness of solutions for a fourth-order boundary value problem," Nonlinear Analysis. Theory, Methods E Applications, vol. 70, no. 10, pp. 3561-3566, 2009.

[5] Y. Li, "Positive solutions of fourth-order boundary value problems with two parameters," Journal of Mathematical Analysis and Applications, vol. 281, no. 2, pp. 477-484, 2003.

[6] Y. Li, "Positive solutions of fourth-order periodic boundary value problems," Nonlinear Analysis. Theory, Methods E Applications, vol. 54, no. 6, pp. 1069-1078, 2003.

[7] X.-L. Liu and W.-T. Li, "Existence and multiplicity of solutions for fourth-order boundary value problems with three parameters," Mathematical and Computer Modelling, vol. 46, no. 3-4, pp. 525-534, 2007.

[8] H. Ma, "Symmetric positive solutions for nonlocal boundary value problems of fourth order," Nonlinear Analysis. Theory, Methods E Applications, vol. 68, no. 3, pp. 645-651, 2008.

[9] R. Ma, "Existence of positive solutions of a fourth-order boundary value problem," Applied Mathematics and Computation, vol. 168, no. 2, pp. 1219-1231, 2005.

[10] C. Pang, W. Dong, and Z. Wei, "Multiple solutions for fourth-order boundary value problem," Journal of Mathematical Analysis and Applications, vol. 314, no. 2, pp. 464-476, 2006. 
[11] Z. Wei and C. Pang, "Positive solutions and multiplicity of fourth-order m-point boundary value problems with two parameters," Nonlinear Analysis. Theory, Methods E Applications, vol. 67, no. 5, pp. 1586-1598, 2007.

[12] Y. Yang and J. Zhang, "Existence of solutions for some fourth-order boundary value problems with parameters," Nonlinear Analysis. Theory, Methods E Applications, vol. 69, no. 4, pp. 1364-1375, 2008.

[13] Q. L. Yao and Z. B. Bai, "Existence of positive solutions of a BVP for $u^{4}(t)-\lambda h(t) f(u(t))=0$," Chinese Annals of Mathematics. Series A, vol. 20, no. 5, pp. 575-578, 1999.

[14] Q. Yao, "Local existence of multiple positive solutions to a singular cantilever beam equation," Journal of Mathematical Analysis and Applications, vol. 363, no. 1, pp. 138-154, 2010.

[15] J. Zhao and W. Ge, "Positive solutions for a higher-order four-point boundary value problem with a p-Laplacian," Computers \& Mathematics with Applications, vol. 58, no. 6, pp. 1103-1112, 2009.

[16] Z. Bai, "Positive solutions of some nonlocal fourth-order boundary value problem," Applied Mathematics and Computation, vol. 215, no. 12, pp. 4191-4197, 2010.

[17] D. J. Guo and V. Lakshmikantham, Nonlinear Problems in Abstract Cones, vol. 5 of Notes and Reports in Mathematics in Science and Engineering, Academic Press, Boston, Mass, USA, 1988. 